\title{
The dynamics of order-order phase separation
}

\author{
K Yamada and S Komura \\ Department of Chemistry, Graduate School of Science and Engineering, Tokyo Metropolitan \\ University, Tokyo 192-0397, Japan \\ E-mail: komura@tmu.ac.jp
}

Received 29 December 2007, in final form 3 March 2008

Published 25 March 2008

Online at stacks.iop.org/JPhysCM/20/155107

\begin{abstract}
In multi-component soft matter, two microphase separated ordered phases such as lamellar and hexagonal phases coexist in equilibrium. We call such a macroscopic phase separation 'order-order phase separation'. Based on a coarse grained single order parameter Ginzburg-Landau free energy, we investigate the dynamics of order-order phase separation. For this purpose, the equilibrium phase diagram is determined in three dimensions, including BCC and gyroid phases. Focusing on the phase separation between the lamellar and hexagonal phases, we study its dynamics using computer simulation in both two and three dimensions. We show that the microphase separation takes place in the early stage and the macrophase separation follows through the breaking and reconnection of microdomains. The observed epitaxial relation between the lamellar and hexagonal phases is compared with an analytical estimate of the interfacial energies of different mutual directions.
\end{abstract}

(Some figures in this article are in colour only in the electronic version)

\section{Introduction}

Phase separation plays an essential role in soft matter, because the resulting interfaces are responsible for the internal mesoscopic structures. The existence of such meso-scale structures is intimately related to non-linear and non-equilibrium behaviors of the materials. In contrast to mixtures of simple liquids, which typically exhibit macrophase separation for certain conditions, systems containing amphiphilic molecules such as block copolymers or surfactants undergo microphase separation at low temperatures. Typical microphase separated structures appear as ordered structures such as lamellar, hexagonal, body-centered-cubic (BCC), and gyroid phases, in which one of the components is periodically arranged in space. In particular, the equilibrium structures in diblock copolymer melts have been extensively studied in the past decades both experimentally and theoretically [1].

In multi-component systems containing amphiphilic molecules, two different ordered phases can generally coexist in thermodynamic equilibrium. We refer to such a macroscopic phase separation and the resulting coexistence between the two ordered phases as 'order-order phase separation'. (One should distinguish 'order-order phase separation' from 'order-order phase transition'. In the latter case, phase transitions from one ordered phase to another ordered phase are considered.) For example, a coexistence between the stripe (two-dimensional lamellar) and hexagonal phases was theoretically predicted for insoluble Langmuir monolayers by Andelman et al [2]. In the experiment, it was found that the stripe (labyrinth) and hexagonal phases coexist in binary mixed monomolecular films containing cholesterol and phospholipid, and that it is possible to observe the phase separation between these two modulated phases [3]. The order-order phase separation between the stripe and hexagonal phases is also anticipated for two-component lipid bilayer membranes in which the composition and the membrane curvature are coupled to each other [4]. In binary mixtures of non-ionic surfactant and water, it is known that the lamellar phase coexists with other mesophases such as the hexagonal or gyroid phases [5, 6].

In different systems, Matsen calculated the binary phase diagrams of $\mathrm{AB}$ diblock copolymer and $\mathrm{A}$ homopolymer having similar degrees of polymerization $[7,8]$. In his work, various phase coexistences among the ordered mesophases have been determined using self-consistent field theory. A more systematic study by changing the ratio of the chain lengths was performed by Janert and Schick $[9,10]$. Moreover, order-order phase separation occurs also for ternary blends of $\mathrm{A}$ and $\mathrm{B}$ homopolymers plus slightly asymmetric $\mathrm{AB}$ diblock 
copolymer [11]. Recently, Mareau et al investigated the interface between the perforated layer phase and gyroid phase by $3 \mathrm{D}$ transmission electron microscopy in a blend of $\mathrm{AB}$ diblock copolymer and homopolymer [12]. In non-equilibrium situations, some groups have identified the coexisting states between distinct structures for block copolymer melts under external fields [13-15].

The previous studies on order-order phase separation have mainly focused on the equilibrium properties and less attention has been paid to its dynamical aspect. In this paper, we discuss the kinetics of order-order phase separation. We shall investigate how the two-phase coexistence is macroscopically achieved starting from a uniformly disordered state. In other words, our primary interest is to clarify the mechanism of a double phase separation, i.e. the competition between the macrophase and microphase separations. A somewhat similar question was raised before for copolymer and homopolymer mixtures by Ohta and Ito, who studied the phase separation dynamics between the lamellar and disordered phases [16].

For this purpose, we employ a modified Ginzburg-Landau free energy, which yields several modulated phases and their coexistences. With the use of a two-mode expansion method, we first construct a mean-field equilibrium phase diagram in three dimensions including the BCC and gyroid phases. Based on the obtained phase diagram, we perform computer simulations of the proposed dynamical equation. In the present study, we mainly focus on the order-order phase separation between the lamellar and hexagonal phases in two dimensions. We also study analytically the equilibrium interfacial profile between the two ordered phases. Attention is paid to the mutual orientation between the lamellar and hexagonal phases in two dimensions. The result will be compared with that from our computer simulation as well as the previous studies on interfaces of modulated phases [17].

This paper is organized as follows. In the next section, we first introduce a single order parameter Ginzburg-Landau free energy functional which is used throughout this paper. Based on this free energy, we present the equilibrium phase diagram obtained by a two-mode expansion method in three dimensions. In section 3, we consider a dynamical model of the order-order phase separation, and perform its computer simulation both in two and three dimensions. Various quantities characterizing the transient structures are calculated in order to clarify typical features of the order-order phase separation. In section 4, we present an analytical calculation of the interface between the lamellar and hexagonal phases for different mutual orientations. Finally, we conclude in section 5.

\section{Model and phase diagram}

One of the simplest free energies that exhibits various modulated phases is given by

$$
F\{\phi(\mathbf{r})\}=\int \mathrm{d} \mathbf{r}\left[2\left(\nabla^{2} \phi\right)^{2}-2(\nabla \phi)^{2}+\frac{\tau}{2} \phi^{2}+\frac{1}{4} \phi^{4}\right],
$$

which was used in [18]. This mean-field free energy is a modified Ginzburg-Landau expansion in the order parameter $\phi(\mathbf{r})=\psi(\mathbf{r})-\psi_{\mathrm{c}}$, where $\psi_{\mathrm{c}}$ is the composition at the critical temperature $T_{\mathrm{c}}$. In the above, $\tau=\left(T-T_{\mathrm{c}}\right) / T_{\mathrm{c}}$ is the reduced temperature, and the coefficients of the other terms are taken to be constant without loss of generality. Spatial modulations are preferred due to the negative coefficient of the gradient square term, while the Laplacian square term plays the role of a homogenizing interaction. The modulation occurs with a dominant wavevector $k^{*}=1 / \sqrt{2}$ due to the competition between these two gradient terms. Such a free energy functional has been used to describe various systems such as diblock copolymers [1], Langmuir films [2], magnetic layers [19] and microemulsions [20]. It should be noticed, however, that the free energy (1) is very generic, and it does not pertain to any specific system.

With the use of equation (1), the phase diagram in two dimensions showing the relative stability of the lamellar (stripe) and hexagonal phase was obtained previously $[2,4,17]$. Here we obtain the phase diagram in three dimensions including the BCC and gyroid phases in addition to these classical phases. For this purpose, we employ a two-mode expansion method and compare between the free energies of different ordered phases [21]. We employ a mean-field approximation, and critical fluctuation corrections are not taken into account $[22,23]$.

In order to express all the ordered structures, we expand $\phi(\mathbf{r})$ as

$$
\phi(\mathbf{r})=\bar{\phi}+2 \sum_{j=1}^{12} a_{j} \cos \left(\mathbf{q}_{j} \cdot \mathbf{r}\right)+2 \sum_{k=1}^{6} b_{k} \cos \left(\mathbf{p}_{k} \cdot \mathbf{r}\right),
$$

where $\bar{\phi}$ is the average composition and $a_{j}$ and $b_{k}$ are real amplitudes. The fundamental reciprocal vectors $\mathbf{q}_{j}$ and $\mathbf{p}_{k}$ are defined by

$$
\begin{aligned}
\mathbf{q}_{1}=C_{Q}(2,-1,1), & \mathbf{q}_{2}=C_{Q}(-2,1,1), \\
\mathbf{q}_{3}=C_{Q}(-2,-1,1), & \mathbf{q}_{4}=C_{Q}(2,1,1), \\
\mathbf{q}_{5}=C_{Q}(-1,-2,1), & \mathbf{q}_{6}=C_{Q}(1,-2,1), \\
\mathbf{q}_{7}=C_{Q}(-1,2,1), & \mathbf{q}_{8}=C_{Q}(1,2,1), \\
\mathbf{q}_{9}=C_{Q}(1,-1,-2), & \mathbf{q}_{10}=C_{Q}(1,1,-2), \\
\mathbf{q}_{11}=C_{Q}(-1,1,-2), & \mathbf{q}_{12}=C_{Q}(-1,-1,-2), \\
\mathbf{p}_{1}=C_{P}(2,2,0), & \mathbf{p}_{2}=C_{P}(2,-2,0), \\
\mathbf{p}_{3}=C_{P}(0,2,2), & \mathbf{p}_{4}=C_{P}(0,-2,2), \\
\mathbf{p}_{5}=C_{P}(2,0,2), & \mathbf{p}_{6}=C_{P}(-2,0,2),
\end{aligned}
$$

with $C_{Q}=Q / \sqrt{6}$ and $C_{P}=P /(2 \sqrt{2})$, where $Q$ and $P$ are the absolute values of $\mathbf{q}_{j}$ and $\mathbf{p}_{k}$, respectively.

Within this expansion, the lamellar phase is given when $a_{1}=A_{\mathrm{L}}$ and all the other amplitudes vanish, whereas the hexagonal phase is given when $a_{3}=-a_{8}=a_{9}=A_{\mathrm{H}}$ and all the other amplitudes are zero. On the other hand, the BCC structure is obtained when $a_{j}=0(j=1, \ldots, 12)$ and $b_{k}=$ $B_{\mathrm{S}}(k=1, \ldots, 6)$. Finally, the gyroid phase is constructed by putting $\left|a_{j}\right|=A_{\mathrm{G}}(j=1, \ldots, 12)$ and $\left|b_{k}\right|=B_{\mathrm{G}}(k=$ $1, \ldots, 6)$, together with the following condition between the absolute values of the reciprocal vectors [21]:

$$
Q^{2}=\frac{3}{4} P^{2} \text {. }
$$




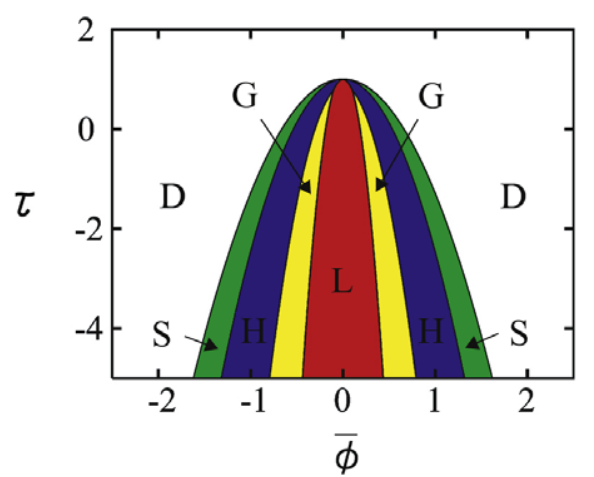

Figure 1. Mean-field phase diagram of the free energy (1) in the $(\bar{\phi}, \tau)$ plane, where $\bar{\phi}$ is the average composition and $\tau$ is the reduced temperature. The red $(\mathrm{L})$, yellow $(\mathrm{G})$, blue $(\mathrm{H})$ and green $(\mathrm{S})$ regions correspond to the lamellar, gyroid, hexagonal and $\mathrm{BCC}$ phases, respectively. The region of the disordered phase is denoted by 'D'. All the lines are first-order transition lines and the critical point is located at $(0,1)$.

Substituting equation (2) into equation (1) and ignoring the higher order harmonics, we can rewrite the free energy in terms of $a_{j}, b_{k}, Q$ and $P$. We leave the full expression to the appendix since it is lengthy. The free energies of the lamellar $\left(F_{\mathrm{L}}\right)$, hexagonal $\left(F_{\mathrm{H}}\right)$, BCC $\left(F_{\mathrm{S}}\right)$, and gyroid phases $\left(F_{\mathrm{G}}\right)$ are obtained as

$$
\begin{aligned}
& F_{\mathrm{L}}=F_{\mathrm{D}}+4 Q^{4} A_{\mathrm{L}}{ }^{2}-4 Q^{2} A_{\mathrm{L}}{ }^{2}+\tau A_{\mathrm{L}}^{2}+3 \bar{\phi}^{2} A_{\mathrm{L}}{ }^{2} \\
& +\frac{3}{2} A_{\mathrm{L}}{ }^{4} \\
& F_{\mathrm{H}}=F_{\mathrm{D}}+12 Q^{4} A_{\mathrm{H}}{ }^{2}-12 Q^{2} A_{\mathrm{H}}{ }^{2}+3 \tau A_{\mathrm{H}}{ }^{2}+9 \bar{\phi}^{2} A_{\mathrm{H}}{ }^{2} \\
& +12 \bar{\phi} A_{\mathrm{H}}{ }^{3}+\frac{45}{2} A_{\mathrm{H}}{ }^{4} \text {, } \\
& F_{\mathrm{S}}=F_{\mathrm{D}}+24 P^{4} B_{\mathrm{S}}{ }^{2}-24 P^{2} B_{\mathrm{S}}{ }^{2}+6 \tau B_{\mathrm{S}}{ }^{2}+18 \bar{\phi}^{2} B_{\mathrm{S}}{ }^{2} \\
& +48 \bar{\phi} B_{\mathrm{S}}{ }^{3}+135 B_{\mathrm{S}}{ }^{4} \text {, } \\
& F_{\mathrm{G}}=F_{\mathrm{D}}+48 Q^{4}{A_{\mathrm{G}}}^{2}+24 P^{2} B_{\mathrm{G}}{ }^{2}-48 Q^{2} A_{\mathrm{G}}{ }^{2}-24 P^{2} B_{\mathrm{G}}{ }^{2} \\
& +12 \tau A_{\mathrm{G}}{ }^{2}+6 \tau B_{\mathrm{G}}{ }^{2}+36 \bar{\phi}^{2} A_{\mathrm{G}}{ }^{2}+18 \bar{\phi}^{2} B_{\mathrm{G}}{ }^{2} \\
& +306 A_{\mathrm{G}}{ }^{4}+216 A_{\mathrm{G}}{ }^{2} B_{\mathrm{G}}{ }^{2}+135 B_{\mathrm{G}}{ }^{4}-48 \bar{\phi} A_{\mathrm{G}}{ }^{3} \\
& +48 \bar{\phi} B_{\mathrm{G}}{ }^{3}-72 \bar{\phi} A_{\mathrm{G}}{ }^{2} B_{\mathrm{G}} \text {, }
\end{aligned}
$$

respectively, where

$$
F_{\mathrm{D}}=\frac{\tau}{2} \bar{\phi}^{2}+\frac{1}{4} \bar{\phi}^{4}
$$

is the free energy of the disordered phase.

First, we compare the above free energies and look for the structure which has the lowest free energy. In other words, we determined the phase boundaries in figure 1 from the crossings of the free energies. Hence we have ruled out the possibility of any phase coexistence between the ordered structures. In figure 1 , the red $(\mathrm{L})$, yellow $(\mathrm{G})$, blue $(\mathrm{H})$ and green $(\mathrm{S})$ regions represent the lamellar, gyroid, hexagonal and BCC phases, respectively. The region of the disordered phase is denoted by ' $\mathrm{D}$ '. All the lines are first-order transition lines. The critical point is located at $(\bar{\phi}, \tau)=(0,1)$, at which a second-order phase transition between the disordered and lamellar phases takes place. The gyroid phase appears
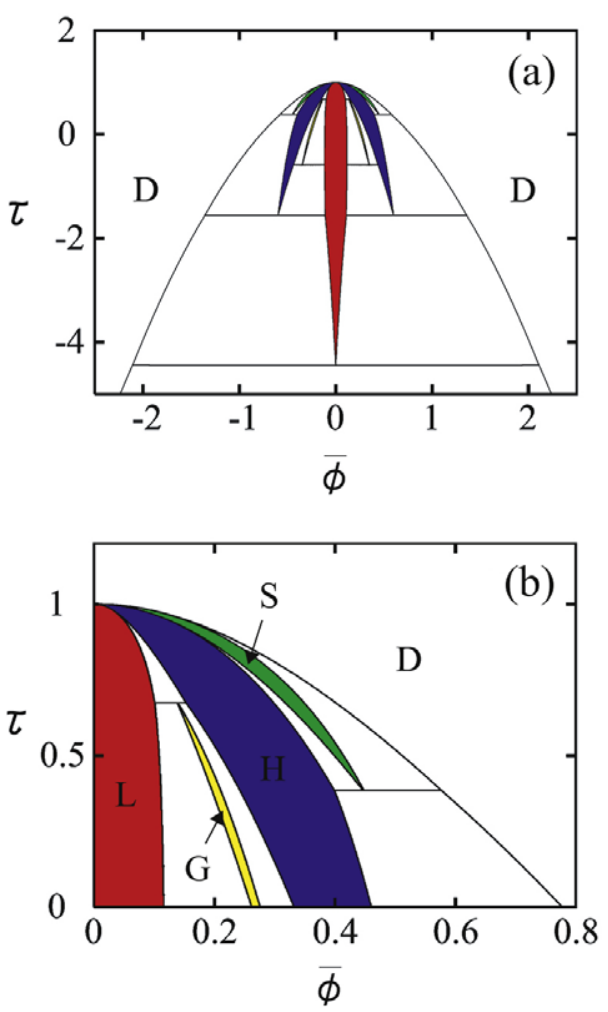

Figure 2. Mean-field phase diagram in the presence of the coexistence regions between the different ordered phases. The red $(\mathrm{L})$, yellow $(\mathrm{G})$, blue $(\mathrm{H})$ and green $(\mathrm{S})$ regions correspond to the lamellar, gyroid, hexagonal and BCC phases, respectively. The white regions are the coexisting regions between the two neighboring phases located on the left- and right-hand sides. (b) The magnification of (a) close to the critical point.

between the lamellar and hexagonal phases, and it ends at $(\bar{\phi}, \tau) \approx( \pm 0.10,0.79)$. This mean-field phase diagram is very similar to that of a diblock copolymer melt obtained by Ginzburg-Landau type theory [21] or self-consistent field theory [24].

Since we are interested in the order-order phase separation, we next calculate the coexistence regions between the different ordered phases using a common tangent construction and the result is presented in figures 2(a) and (b) (the latter is a magnification of the former close to the critical point). The white regions are the coexisting regions between the two neighboring phases located on the left- and right-hand sides. The lamellar-hexagonal coexistence ends at $\tau \approx 0.67$, and it starts again at $\tau \approx-0.60$ and ends at $\tau \approx-1.56$. The lamellar-gyroid and gyroid-hexagonal coexistences occurs for $-0.60 \leqslant \tau \leqslant 0.67$. The hexagonalBCC coexistence ends at $\tau \approx 0.38$. Although a phase diagram with coexistence regions has been obtained before in $2 \mathrm{D}$ systems in the absence of the BCC and gyroid phases [4, 17], the corresponding phase diagram in $3 \mathrm{D}$ has not yet been calculated. It should be noted, however, that the binodal lines between the lamellar and hexagonal phases coincide both in $2 \mathrm{D}$ and $3 \mathrm{D}$ systems. We shall further investigate this lamellar-hexagonal coexistence both by computer simulation and analytical treatment. 
(a)

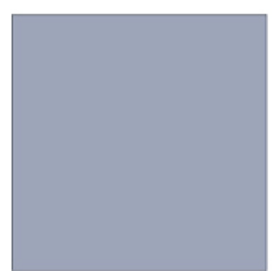

(b)

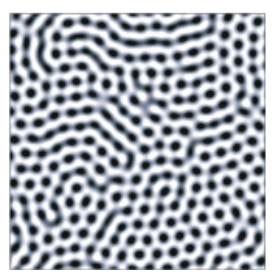

(c)

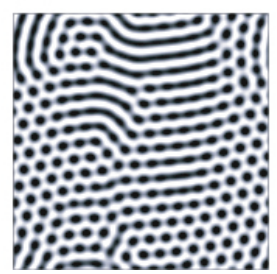

(d)

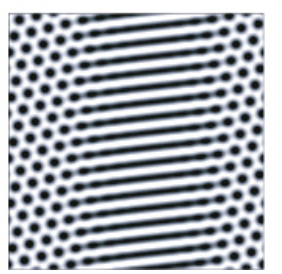

Figure 3. Time evolution of phase separation pattern for $\bar{\phi}=0.1$ and $\tau=0.8$ in two dimensions. (a) $t=0$, (b) $t=150$, (c) $t=500$, (d) $t=5000$.

(a)

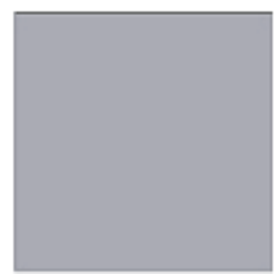

(b)

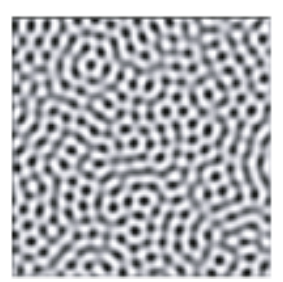

(c)

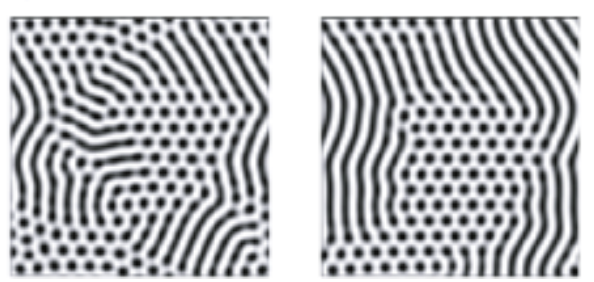

Figure 4. Time evolution of phase separation pattern for $\bar{\phi}=0.12$ and $\tau=0.7$ in two dimensions. (a) $t=0$, (b) $t=70$, (c) $t=500$, (d) $t=5000$.

\section{Dynamics}

\subsection{Dynamical model and simulation method}

In this section, we discuss the dynamics of order-order phase separation by means of numerical simulations. For the time evolution of the relative composition $\phi$, which is a conserved quantity, we consider the following equation:

$$
\begin{aligned}
\frac{\partial \phi}{\partial t} & =\nabla^{2} \frac{\delta F}{\delta \phi} \\
& =\nabla^{2}\left[4 \nabla^{4} \phi+4 \nabla^{2} \phi+\tau \phi+\phi^{3}\right] .
\end{aligned}
$$

For simplicity, the kinetic coefficient is taken to be unity. In the present dynamical model, we neglect both the hydrodynamic effect and thermal fluctuations.

In the numerical simulation, we have used the implicit method with the FFTW algorithm. Compared to the Euler's method, this algorithm is useful in solving differential equations with higher order derivatives such as equation (10). We have employed the periodic boundary condition and the simulation starts from a disordered state with a small random noise around the average composition $\bar{\phi}$. Hereafter we focus on the coexistence region between the lamellar and hexagonal phases (see figure 2(b)). We performed numerical simulations both in two and three dimensions, although the latter result is still preliminary. In $2 \mathrm{D}$ simulations, the mesh number is $256 \times 256$, the time increment is $\Delta t=0.01$, and the space increment is $\Delta x=0.59$. In $3 \mathrm{D}$ simulation, on the other hand, the mesh number is $64 \times 64 \times 64$, the time increment is $\Delta t=0.01$, and the space increment is $\Delta x=0.65$.

\section{2. $2 D$ simulation}

In figures 3 and 4 we show typical time evolutions of the order parameter $\phi$ in two dimensions when $(\bar{\phi}, \tau)=(0.1,0.8)$ and $(0.12,0.7)$, respectively. Notice that these points are located inside the coexistence region between the lamellar and hexagonal phases in figure 2(b). In the early stage, many circular spots start to appear simultaneously, as shown in figure 3 (b) at $t=150$. Some of the spots are separated while the others are connected to each other to form stripes. Then we see in figure 3(c) that the breaking and reconnection of the stripes take place so that large domains are gradually formed, although it is still hard to identify clear domains. Finally, we obtain the coexistence between the lamellar and hexagonal phases as shown in figure 3(d). Because this pattern persists until $t=20000$, we believe that the system has reached almost the equilibrium (or at least metastable) state. A qualitatively similar time evolution can be observed in figure 4 with a different choice of parameters. Hence we expect that the kinetics of order-order phase separation between the lamellar and hexagonal phases is rather universal, at least in two dimensions.

The above sequence of patterns tells us that the orderorder phase separation initially starts with a microphase separation, and a macrophase separation follows later through the rearrangement of microdomains. To see this behavior more quantitatively, we present in figure 5 the Fourier transformed patterns of figure 3 in the two-dimensional reciprocal space. As seen in figure 5(b), a circular ring pattern is seen in the early stage at $t=150$. This means that the structure is almost isotropic on average and is characterized by the typical wavelength $k^{*}$. Hence the microphase separation dominates in this time region. Then the six spots (Bragg peaks) become pronounced in the course of time as in figure 5(c), indicating that the structure becomes anisotropic with sixfold symmetry. The Fourier pattern of the final state is given by figure $5(\mathrm{~d})$, which is very similar to that of the hexagonal structure. However, it should be noted that the two peaks 
(a)

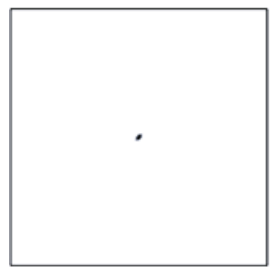

(b)

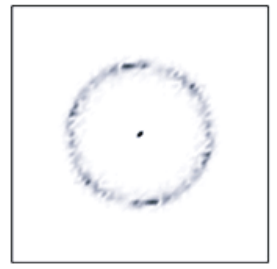

(c)

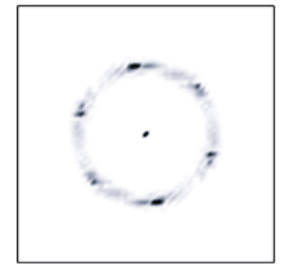

(d)

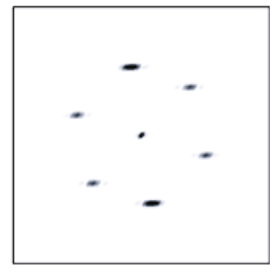

Figure 5. Two-dimensional Fourier patterns of figure 3 in the reciprocal space. (a) $t=0$, (b) $t=150$, (c) $t=500$, (d) $t=5000$.
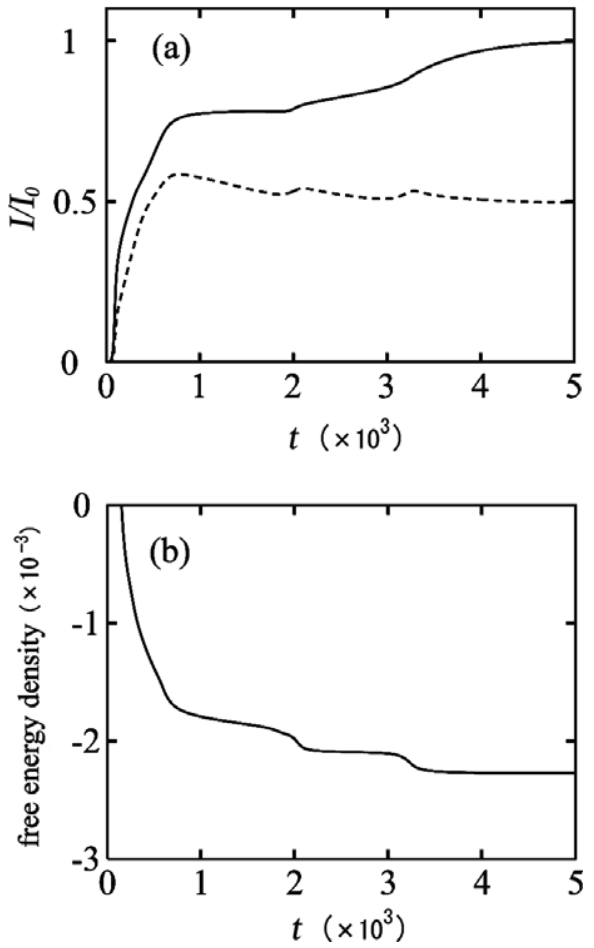

Figure 6. (a) Time evolution of the Bragg peak intensities for $\bar{\phi}=0.1$ and $\tau=0.8$, as shown in figure 5. The vertical axis is the peak intensity $I$ normalized by its value at $t=20000$, denoted as $I_{0}$. The solid line corresponds to the peak of the lamellar pattern while the dashed line is that of the hexagonal pattern. (b) Time evolution of the free energy density for the same parameter values.

corresponding to the lamellar pattern (the upper and lower spots) have stronger intensities than the other four peaks. This means that there is an epitaxial relation between the lamellar and hexagonal phases in the macroscopically phase separated system. A similar epitaxial relation has been identified in the kinetic pathways of order-order transitions in diblock copolymer melt [25-27].

In figure 6(a), we plot the time evolution of the Bragg peak intensities $I$ in figure 5. We follow the peak intensity of the upper spot corresponding to the lamellar structure (solid line), and that of the other four peaks reflecting the hexagonal structure (dashed line). Here the respective peak intensities are normalized by the lamellar peak value $I_{0}$ at $t=20000$. Both intensities increase almost in the same manner up to $t \approx 100$ and then they grow separately. Some cusps in the curves reflect breaking and reconnection of microdomains as explained above. In the late stage, the lamellar peak intensity is twice as large as that of the hexagonal phase. This ratio is dependent on the average composition $\bar{\phi}$. According to the lever rule, the lamellar peak intensity will increase as $\bar{\phi}$ is decreased in the coexistence region. Figure 6(b) is the time evolution of the free energy density. It decreases monotonically as a function of time to reach the final structure.

In order to further quantify the evolution of the phase separated patterns, we calculate the so called Minkowski functionals. These quantities are used in digital picture analysis for the characterization of black-and-white discrete images. For the details of Minkowski functionals, see [28-30]. In the patterns of figure 3 , we first reset the gray value of each lattice to either white or black depending on whether the original value is larger or lower than the threshold value $\phi=0$, respectively. (The result is insensitive to the choice of the threshold value as long as $\phi$ is close to zero.) Then three quantities are calculated: (i) relative white area $v$, (ii) length of the boundary line $s$ between black and white regions, and (iii) Euler characteristic $\chi$, which measures the connectivity of the black and white regions [28]. These quantities in the simulation of figure 3 are plotted in figure 7 as a function of time. The relative white area $v$ increases rapidly in the early stage and saturates after $t \approx$ 800 , as seen in figures 7 (a) and (b). The length of the boundary line $s$ takes a maximum value at around $t \approx 120$, when many circular domains are formed. In figures 7(e) and (f), the absolute value of the Euler characteristic $\chi$ takes large values in the early stage, since many circular domains appear. Then it decreases because they connect to form long stripes. These time evolutions of Minkowski functionals are consistent with the analysis of the Fourier patterns.

\section{3. $3 D$ simulation}

Based on the phase diagram of figure 2(b), we have also carried out the numerical simulation in three dimensions. In figure 8 , we show the time evolution of the $3 \mathrm{D}$ pattern for $(\bar{\phi}, \tau)=(0.1,0.8)$. Notice that this choice of the parameters is the same as that in figure 3. As mentioned before, the coexistence lines between the lamellar and hexagonal phases in this region are identical between the $2 \mathrm{D}$ and $3 \mathrm{D}$ cases. The surfaces in figure 8 represent the iso-planes at $\phi=-0.2$ and we do not see any pattern in figure 8(a). In the early stage, a random network appears, as seen in figure 8(b). This may correspond to the phase separated patterns observed 

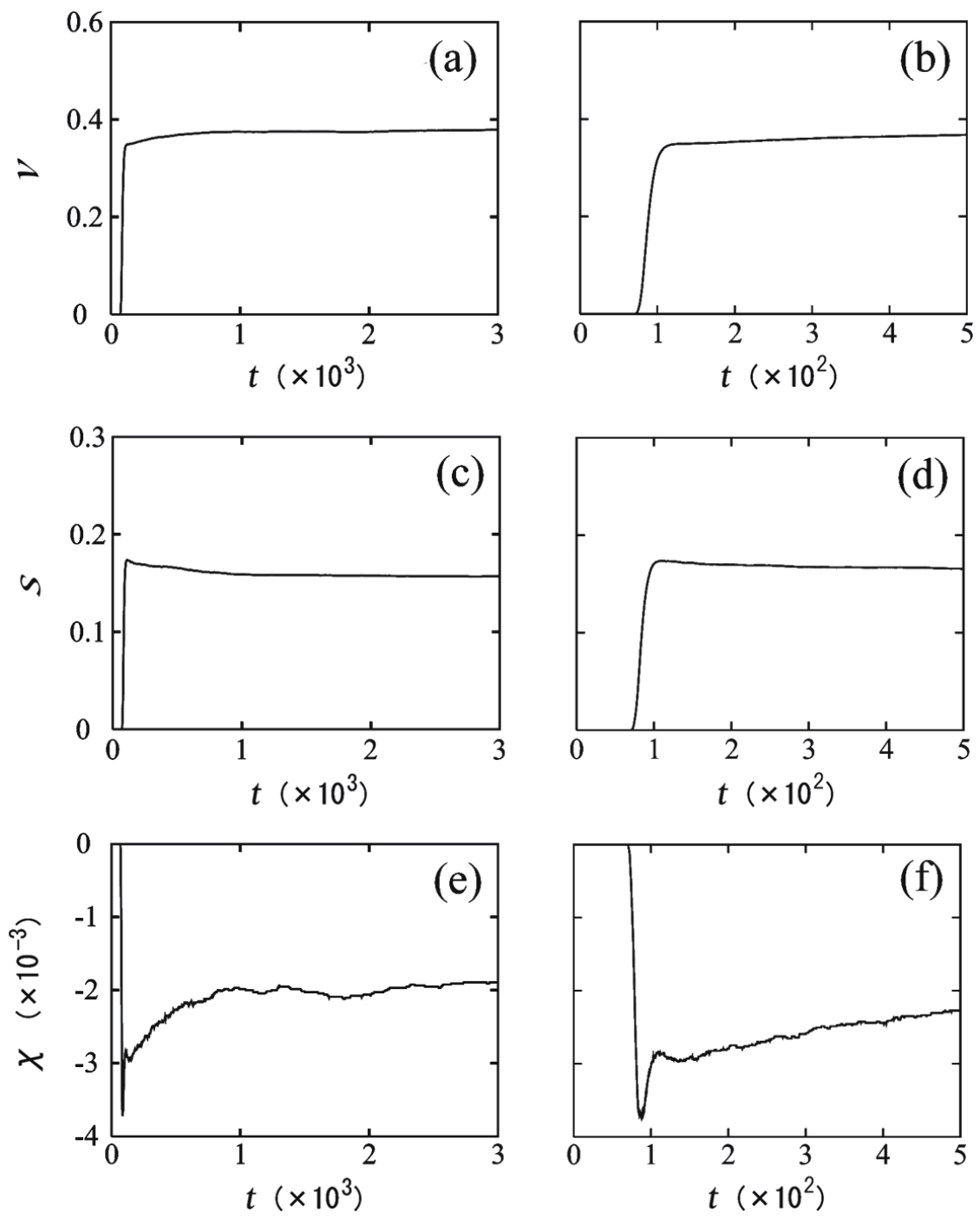

Figure 7. Time evolution of Minkowski functionals for $\bar{\phi}=0.1$ and $\tau=0.8$, as shown in figure 3. (a), (b) The relative black area $v$, (c), (d) the length of the boundary line $s$ between black and white regions, (e), (f) the Euler characteristic $\chi$. (b), (d) and (f) are the expansions of the small-time regions of (a), (c) and (e), respectively.

(a)

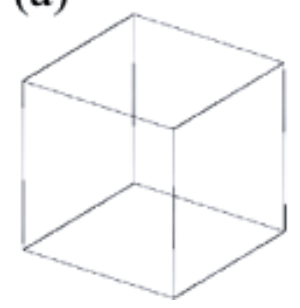

(b)

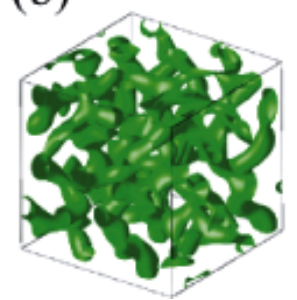

(c)

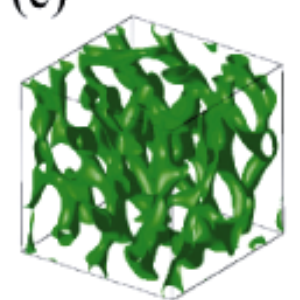

(d)

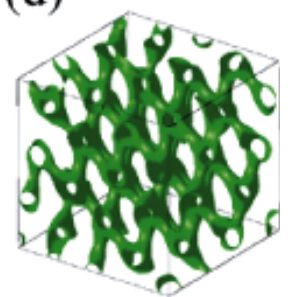

Figure 8. Time evolution of phase separation pattern for $\bar{\phi}=0.1$ and $\tau=0.8$ in three dimensions. (a) $t=0$, (b) $t=150$, (c) $t=1000$, (d) $t=5000$. The surfaces represent the iso-planes at $\phi=-0.2$.

in the 2D simulations such as in figures $3(\mathrm{~b})$ and $4(\mathrm{~b})$. Through the breaking and reconnection of the network, we finally obtain an almost periodic structure as presented in figure $8(d)$.

Due to the small system size, we could not observe any macroscopic phase separation between the lamellar and hexagonal phases in this simulation. Instead, we obtained the Bragg peak intensities of figure 8(d), as presented in table 1 . We show here only the peaks whose intensities are larger than 0.02. It should be noted that this structure was not taken into account when we calculated the phase diagram in section 2 . 
Table 1. The Bragg peak intensities of the structure shown in figure 8(d). We list only the peaks whose intensities are larger than 0.02 .

\begin{tabular}{ll}
\hline Reciprocal vectors & Intensity \\
\hline$(-2,-1,4)$ & 0.122064 \\
$(-3,3,2)$ & 0.119915 \\
$(-1,2,4)$ & 0.071968 \\
$(-4,0,2)$ & 0.066277 \\
$(1,-4,2)$ & 0.062359 \\
$(-4,2,1)$ & 0.056203 \\
$(-3,-2,3)$ & 0.048956 \\
$(2,4,1)$ & 0.039712 \\
$(3,0,3)$ & 0.032639 \\
$(2,-3,3)$ & 0.023958 \\
\hline
\end{tabular}

\section{Interface between the lamellar and hexagonal phases}

In this section, we study the mutual orientation between the lamellar and the hexagonal phases at the interface when these two phases coexist in equilibrium. We shall determine analytically the most stable interfacial profile between the two phases. For simplicity, we compare between the four different interfaces shown in figure 9, where we choose the horizontal and vertical axes as the $x$ - and $y$-directions, respectively. Here the directions parallel to the stripe and the interface make angles (a) 0 , (b) $\pi / 3$, (c) $\pi / 2$, and (d) $\pi / 6$, respectively.

In the cases of figures 9 (a) and (b), the spatially varying order parameter $\phi(\mathbf{r})$ can be expressed as

$$
\phi(\mathbf{r})=\bar{\phi}(x)+2 \sum_{i=1}^{3} c_{i}(x) \cos \left(\mathbf{k}_{i} \cdot \mathbf{r}\right),
$$

where the three reciprocal vectors are defined by

$$
\begin{gathered}
\mathbf{k}_{1}=k^{*}(1,0), \quad \mathbf{k}_{2}=k^{*}(1 / 2, \sqrt{3} / 2), \\
\mathbf{k}_{3}=k^{*}(1 / 2,-\sqrt{3} / 2) .
\end{gathered}
$$

Notice here that the average composition $\bar{\phi}(x)$ and the amplitudes $c_{i}(x)$ are functions of $x$. Substitution of equation (11) into equation (1) yields the equilibrium free energy in terms of $\bar{\phi}(x), c_{i}(x)$, and $k^{*}$. In the calculation, we use an approximation such that all the higher order harmonics are ignored, which is valid in the weak segregation limit. For example, the second term of equation (1) becomes

$$
\begin{aligned}
& \int \mathrm{d} \mathbf{r}(\nabla \phi)^{2}=L_{y} \int \mathrm{d} x\left(\frac{\partial \bar{\phi}}{\partial x}\right)^{2}+2 L_{y} \int \mathrm{d} x \sum_{i=1}^{3}\left[\left(\frac{\partial c_{i}}{\partial x}\right)^{2}\right. \\
& \left.+k^{* 2} c_{i}^{2}\right]+4 L_{y} \int \mathrm{d} x\left[\frac{\partial \bar{\phi}}{\partial x} \frac{\partial c_{1}}{\partial x}+\frac{\partial c_{2}}{\partial x} \frac{\partial c_{3}}{\partial x}+\frac{k^{* 2}}{2} c_{2} c_{3}\right] \\
& \quad \times \cos \left(\mathbf{k}_{1} \cdot \mathbf{r}\right)+4 L_{y} \int \mathrm{d} x\left[-k^{*} c_{1} \frac{\partial \bar{\phi}}{\partial x}-\frac{k^{*}}{2} c_{2} \frac{\partial c_{3}}{\partial x}\right. \\
& \left.-\frac{k^{*}}{2} c_{3} \frac{\partial c_{2}}{\partial x}\right] \sin \left(\mathbf{k}_{1} \cdot \mathbf{r}\right),
\end{aligned}
$$

where we have assumed the periodic boundary condition in the $y$-direction, and $L_{y}$ is the system size in the $y$-direction.
Introducing a phase factor $x_{0}$ and writing as $\mathbf{k}_{1} \cdot \mathbf{r}=k^{*}\left(x-x_{0}\right)$, we carry out the integration by parts. The other terms of the free energy are not presented here because they are lengthy.

In the case of configuration (a), we postulate that the amplitudes $c_{i}(x)$ and the average composition $\bar{\phi}(x)$ are expressed as

$$
\begin{gathered}
c_{1}(x)=\frac{1}{2}\left[1+\tanh \left(\frac{x}{\xi}\right)\right] a_{\mathrm{H}}+\frac{1}{2}\left[1-\tanh \left(\frac{x}{\xi}\right)\right] a_{\mathrm{L}} \\
c_{i}(x)=\frac{1}{2}\left[1+\tanh \left(\frac{x}{\xi}\right)\right] a_{\mathrm{H}} \quad(i=2,3), \\
\bar{\phi}(x)=\frac{1}{2}\left[1+\tanh \left(\frac{x}{\xi}\right)\right] \bar{\phi}_{\mathrm{H}}+\frac{1}{2}\left[1-\tanh \left(\frac{x}{\xi}\right)\right] \bar{\phi}_{\mathrm{L}}
\end{gathered}
$$

where $a_{\mathrm{H}}$ and $a_{\mathrm{L}}$ represent the equilibrium amplitudes of the hexagonal and lamellar phases, while $\bar{\phi}_{\mathrm{H}}$ and $\bar{\phi}_{\mathrm{L}}$ are the average compositions in these phases, respectively. The length $\xi$ determines the interfacial width, which is assumed to be much smaller than the spatial period of the modulated structures, i.e. $k^{*} \xi \ll 1$. In the case of (b), on the other hand, the amplitudes $c_{i}(x)$ can be written as

$$
\begin{gathered}
c_{2}(x)=\frac{1}{2}\left[1+\tanh \left(\frac{x}{\xi}\right)\right] a_{\mathrm{H}}+\frac{1}{2}\left[1-\tanh \left(\frac{x}{\xi}\right)\right] a_{\mathrm{L}}, \\
c_{i}(x)=\frac{1}{2}\left[1+\tanh \left(\frac{x}{\xi}\right)\right] a_{\mathrm{H}} \quad(i=1,3),
\end{gathered}
$$

and $\bar{\phi}(x)$ is identical to equation (16).

Next we perform the variable transformation $x / \xi \rightarrow x$. From the assumption $k^{*} \xi \ll 1$, we see that the third-order terms in $\xi^{-1}$ are dominant in the free energy functional. Hence, in the cases of figures 9(a) and (b), the free energy can be approximated as

$$
\begin{aligned}
F\left\{\bar{\phi}(x), c_{i}(x)\right\} \approx 2 L_{y} \xi^{-3} \int \mathrm{d} x\left[\frac{\partial^{2} \bar{\phi}}{\partial x^{2}}+2 \sum_{i=1}^{3}\left(\frac{\partial^{2} c_{i}}{\partial x^{2}}\right)^{2}\right] \\
+8 L_{y} \xi^{-3} \int \mathrm{d} x\left[-\frac{\partial^{2} \bar{\phi}}{\partial x^{2}} \frac{\partial^{2} c_{1}}{\partial x^{2}}-\frac{\partial^{2} c_{2}}{\partial x^{2}} \frac{\partial^{2} c_{3}}{\partial x^{2}}-2 \frac{\partial c_{1}}{\partial x} \frac{\partial^{3} \bar{\phi}}{\partial x^{3}}\right. \\
\left.-\frac{\partial c_{2}}{\partial x} \frac{\partial^{3} c_{3}}{\partial x^{3}}-\frac{\partial c_{3}}{\partial x} \frac{\partial^{3} c_{2}}{\partial x^{3}}\right] \cos \left(k^{*} x_{0}\right)
\end{aligned}
$$

Using equations (14)-(16), we obtain for case (a) as

$$
\begin{aligned}
F_{\mathrm{a}} \approx & 2 L_{y} \xi^{-3} \int \mathrm{d} x\left(\bar{\phi}_{\mathrm{H}}^{2}-2 \bar{\phi}_{\mathrm{H}} \bar{\phi}_{\mathrm{L}}+\bar{\phi}_{\mathrm{L}}^{2}+6 a_{\mathrm{H}}^{2}-4 a_{\mathrm{H}} a_{\mathrm{L}}\right. \\
& \left.+2 a_{\mathrm{L}}^{2}\right) \frac{\sinh ^{2} x}{\cosh ^{6} x}+8 L_{y} \xi^{-3} \cos \left(k^{*} x_{0}\right) \\
& \times \int \mathrm{d} x\left[\left\{-4\left(\bar{\phi}_{\mathrm{H}}-\bar{\phi}_{\mathrm{L}}\right)\left(a_{\mathrm{H}}-a_{\mathrm{L}}\right)-4 a_{\mathrm{H}}^{2}\right\} \frac{\sinh ^{2} x}{\cosh ^{6} x}\right. \\
& \left.+\left\{\left(\bar{\phi}_{\mathrm{H}}-\bar{\phi}_{\mathrm{L}}\right)\left(a_{\mathrm{H}}-a_{\mathrm{L}}\right)+a_{\mathrm{H}}^{2}\right\} \frac{1}{\cosh ^{4} x}\right] \\
\approx & \frac{8}{15} L_{y} \xi^{-3}\left(\bar{\phi}_{\mathrm{H}}^{2}-2 \bar{\phi}_{\mathrm{H}} \bar{\phi}_{\mathrm{L}}+\bar{\phi}_{\mathrm{L}}^{2}+6 a_{\mathrm{H}}^{2}-4 a_{\mathrm{H}} a_{\mathrm{L}}+2 a_{\mathrm{L}}^{2}\right) \\
& +\frac{32}{15} L_{y} \xi^{-3} \cos \left(k^{*} x_{0}\right)\left[\left(\bar{\phi}_{\mathrm{H}}-\bar{\phi}_{\mathrm{L}}\right)\left(a_{\mathrm{H}}-a_{\mathrm{L}}\right)+a_{\mathrm{H}}^{2}\right] .
\end{aligned}
$$


(a)

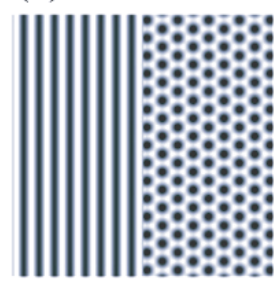

(b)

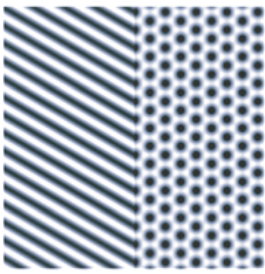

(c)

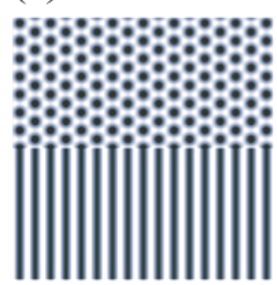

(d)

Figure 9. Four possible interfaces between the coexisting lamellar and hexagonal phases.

Here we considered that the integral ranges from $-\infty$ to $\infty$, and used the following integrals:

$$
\begin{aligned}
& \int_{-\infty}^{\infty} \mathrm{d} x \frac{\sinh ^{2} x}{\cosh ^{6} x}=\frac{4}{15} \\
& \int_{-\infty}^{\infty} \mathrm{d} x \frac{1}{\cosh ^{4} x}=\frac{4}{3} .
\end{aligned}
$$

Similarly, we obtain the free energy for case (b) as

$$
\begin{aligned}
F_{\mathrm{b}} \approx & \frac{8}{15} L_{y} \xi^{-3}\left(\bar{\phi}_{\mathrm{H}}^{2}-2 \bar{\phi}_{\mathrm{H}} \bar{\phi}_{\mathrm{L}}+\bar{\phi}_{\mathrm{L}}^{2}+6 a_{\mathrm{H}}^{2}-4 a_{\mathrm{H}} a_{\mathrm{L}}+2 a_{\mathrm{L}}{ }^{2}\right) \\
& +\frac{32}{15} L_{y} \xi^{-3} \cos \left(k^{*} x_{0}\right)\left(\bar{\phi}_{\mathrm{H}}-\bar{\phi}_{\mathrm{L}}+a_{\mathrm{H}}-a_{\mathrm{L}}\right) a_{\mathrm{H}} .
\end{aligned}
$$

In the cases of figures 9(c) and (d), the amplitudes vary along the $y$-direction and are given by

$$
\begin{gathered}
c_{1}(y)=\frac{1}{2}\left[1+\tanh \left(\frac{y}{\xi}\right)\right] a_{\mathrm{H}}+\frac{1}{2}\left[1-\tanh \left(\frac{y}{\xi}\right)\right] a_{\mathrm{L}}, \\
c_{i}(y)=\frac{1}{2}\left[1+\tanh \left(\frac{y}{\xi}\right)\right] a_{\mathrm{H}} \quad(i=2,3),
\end{gathered}
$$

and

$$
\begin{gathered}
c_{2}(y)=\frac{1}{2}\left[1+\tanh \left(\frac{y}{\xi}\right)\right] a_{\mathrm{H}}+\frac{1}{2}\left[1-\tanh \left(\frac{y}{\xi}\right)\right] a_{\mathrm{L}}, \\
c_{i}(y)=\frac{1}{2}\left[1+\tanh \left(\frac{y}{\xi}\right)\right] a_{\mathrm{H}} \quad(i=1,3),
\end{gathered}
$$

respectively. In both cases, the mean composition $\bar{\phi}(y)$ is also a function of $y$, and is given by

$$
\bar{\phi}(y)=\frac{1}{2}\left[1+\tanh \left(\frac{y}{\xi}\right)\right] \bar{\phi}_{\mathrm{H}}+\frac{1}{2}\left[1-\tanh \left(\frac{y}{\xi}\right)\right] \bar{\phi}_{\mathrm{L}} .
$$

By repeating similar calculations to the cases of (a) and (b), the free energy of cases (c) and (d) is obtained as

$$
\begin{aligned}
& F\left\{\bar{\phi}(y), c_{i}(y)\right\} \approx 2 L_{x} \xi^{-3} \int \mathrm{d} y\left[\left(\frac{\partial^{2} \bar{\phi}}{\partial y^{2}}\right)^{2}\right. \\
& \left.+2 \sum_{i=1}^{3}\left(\frac{\partial^{2} c_{i}}{\partial y^{2}}\right)^{2}\right],
\end{aligned}
$$

where $L_{x}$ is the system size in the $x$-direction. Finally, the free energies of the interfaces (c) and (d) are given by

$$
\begin{aligned}
F_{\mathrm{c}} \approx & F_{\mathrm{d}} \approx \frac{8}{15} L_{x} \xi^{-3}\left(\bar{\phi}_{\mathrm{H}}^{2}-2 \bar{\phi}_{\mathrm{H}} \bar{\phi}_{\mathrm{L}}+\bar{\phi}_{\mathrm{L}}^{2}+6 a_{\mathrm{H}}^{2}\right. \\
& \left.-4 a_{\mathrm{H}} a_{\mathrm{L}}+2 a_{\mathrm{L}}^{2}\right) .
\end{aligned}
$$

For the parameter values $\bar{\phi}=0.1$ and $\tau=0.8$, as we chose in the $2 \mathrm{D}$ and $3 \mathrm{D}$ simulations, we can numerically estimate $a_{\mathrm{H}} \approx-0.12886, a_{\mathrm{L}} \approx-0.24346, \bar{\phi}_{\mathrm{H}} \approx 0.113$ and $\bar{\phi}_{\mathrm{L}} \approx 0.086$ from the phase diagram in figure 2 . With these values, one can evaluate the minimum of the above free energies as $F_{\mathrm{a}} \approx 0.0078 L_{y} \xi^{-3}$ when $k^{*} x_{0}=\pi, F_{\mathrm{b}} \approx$ $0.0109 L_{y} \xi^{-3}$ when $k^{*} x_{0}=0$, and $F_{\mathrm{c}} \approx F_{\mathrm{d}} \approx 0.0498 L_{x} \xi^{-3}$. The difference between cases (c) and (d) appears only in the order of $\xi^{-1}$, and it is estimated as $F_{\mathrm{c}}-F_{\mathrm{d}} \approx 0.014 L_{x} \xi^{-1}>0$. Summarizing all these results, we find that $F_{\mathrm{a}}<F_{\mathrm{b}}<F_{\mathrm{d}}<$ $F_{\mathrm{c}}$ holds when $L_{x}=L_{y}$. Hence the case of figure 9(a) is most stable among the other orientations within the present approximation.

In the 2D simulation results of figures 3(d) and 4(d), we mostly observe the interfaces corresponding to the case of figure 9(b) rather than case (a). Hence it is likely that the system is trapped in a metastable state in the simulation. One of the possibilities to avoid such a metastable state in the simulation is to include a thermal noise term in equation (10). However, we leave this possibility as our future task, because it is expensive in computer time. It could be also possible that the condition $k^{*} \xi \ll 1$ which is assumed in the above calculation may not be satisfied in the simulation. We also note that the estimated energy difference between cases (a) and (b) is rather small, at least compared to either case (c) or (d).

The interface between the lamellar and hexagonal phases was previously studied by Netz et al based on a free energy similar to equation (1) [17]. They investigated the effect of temperature on the interfacial energy using a numerical conjugate-gradient method. In their work, the mutual orientation between the two phases was assumed to be that of figure 9(a). This is in agreement with our analytical argument presented above. We note that the interface of figure 9(a) was indeed observed in some amphiphilic systems [31, 32].

\section{Conclusion}

In the present paper, we have investigated both the equilibrium and dynamical properties of order-order phase separation. Based on a single order parameter Ginzburg-Landau free energy, we first obtained the phase diagram in three dimensions within the two-mode expansion method. Various phase coexistences between the different ordered phases have been identified, including the BCC and gyroid phases. Using computer simulation, we have next focused on the phase separation kinetics between the lamellar and hexagonal 
phases both in two and three dimensions. We find that the microphase separation takes place in the early stage and the macrophase separation follows through the breaking and reconnection of microdomains. This fact has also been confirmed by analyzing the time evolutions of the Fourier patterns and various Minkowski functionals. Finally, we have analytically determined the most stable orientation at the interface between the lamellar and hexagonal phases.

Further study of the order-order phase separation is now in progress. In this paper we have discussed only the dynamics of the lamellar-hexagonal phase separation. Certainly other combinations of phase coexistence are possible in 3D systems, including the BCC and gyroid phases (see figure 2). We are investigating the kinetics of other types of order-order phase separation as well as the equilibrium interfacial properties. It is also interesting to see how one coexistence state between the ordered phases transforms to the other coexistence state by, for example, lowering the temperature. In real systems, critical fluctuations can affect the phase behavior close to the critical point [22, 23]. Investigation to see how the various phase coexistences are modified by critical fluctuations is left for a future problem.

\section{Acknowledgments}

This work is supported by KAKENHI (Grant-in-Aid for Scientific Research) on Priority Area 'Soft matter physics' and grant No 18540410 from the Ministry of Education, Culture, Sports, Science and Technology of Japan.

\section{Appendix. General form of the free energy}

Substituting equation (2) into equation (1) and ignoring the higher order harmonics, we can rewrite the free energy in terms of $a_{j}, b_{k}, Q$ and $P$ as follows:

$$
\begin{aligned}
F= & \left(4 Q^{4}-4 Q^{2}+\tau+3 \bar{\phi}^{2}\right) \sum_{j=1}^{12} a_{j}^{2} \\
& +\left(4 P^{4}-4 P^{2}+\tau+3 \bar{\phi}^{2}\right) \sum_{k=1}^{6} b_{k}^{2} \\
& +3\left(\sum_{j=1}^{12} a_{j}^{2}+\sum_{k=1}^{6} b_{k}^{2}\right)^{2}-\frac{3}{2} \sum_{j=1}^{12} a_{j}^{4}-\frac{3}{2} \sum_{k=1}^{6} b_{k}^{4} \\
& +12 \bar{\phi}\left(a_{1} a_{3} b_{4}+a_{1} a_{7} a_{12}+a_{2} a_{4} b_{3}+a_{2} a_{6} a_{10}+a_{3} a_{8} a_{9}\right. \\
& +a_{4} a_{5} a_{11}+a_{5} a_{7} b_{6}+a_{6} a_{8} b_{5}+a_{9} a_{11} b_{2}+a_{10} a_{12} b_{1} \\
& \left.+b_{1} b_{3} b_{6}+b_{1} b_{4} b_{5}+b_{2} b_{3} b_{5}+b_{2} b_{4} b_{6}\right) \\
& +12\left(a_{1}^{2} b_{2} b_{5}+a_{2}^{2} b_{2} b_{6}+a_{3}^{2} b_{1} b_{6}+a_{4}^{2} b_{1} b_{5}+a_{5}^{2} b_{1} b_{4}\right. \\
& +a_{6}^{2} b_{2} b_{4}+a_{7}^{2} b_{2} b_{3}+a_{8}^{2} b_{1} b_{3}+a_{9}^{2} b_{3} b_{6} \\
& +a_{10}{ }^{2} b_{4} b_{6}+a_{11}{ }^{2} b_{4} b_{5}+a_{12}{ }^{2} b_{3} b_{5}+a_{1} a_{2} a_{3} a_{4} \\
& +a_{1} a_{2} a_{5} a_{8}+a_{1} a_{2} a_{6} a_{7}+a_{1} a_{3} b_{1} b_{2}+a_{1} a_{3} b_{1} b_{5} \\
& +a_{1} a_{3} b_{2} b_{6}+a_{1} a_{3} b_{5} b_{6}+a_{1} a_{4} a_{9} a_{10}+a_{1} a_{4} a_{11} a_{12} \\
& +a_{1} a_{5} a_{10} b_{2}+a_{1} a_{5} a_{10} b_{5}+a_{1} a_{5} a_{12} b_{6}+a_{1} a_{6} a_{9} b_{3} \\
& +a_{1} a_{6} a_{9} b_{6}+a_{1} a_{6} a_{11} b_{2}+a_{1} a_{6} a_{11} b_{4}+a_{1} a_{6} a_{11} b_{5}
\end{aligned}
$$

$$
\begin{aligned}
& +a_{1} a_{7} a_{10} b_{1}+a_{1} a_{8} a_{9} b_{4}+a_{1} a_{8} a_{11} b_{1}+a_{1} a_{8} a_{11} b_{3} \\
& +a_{2} a_{3} a_{9} a_{10}+a_{2} a_{3} a_{11} a_{12}+a_{2} a_{4} b_{1} b_{2}+a_{2} a_{4} b_{1} b_{6} \\
& +a_{2} a_{4} b_{2} b_{5}+a_{2} a_{4} b_{5} b_{6}+a_{2} a_{5} a_{9} b_{1}+a_{2} a_{5} a_{9} b_{4} \\
& +a_{2} a_{5} a_{11} b_{3}+a_{2} a_{6} a_{12} b_{1}+a_{2} a_{7} a_{9} b_{2}+a_{2} a_{7} a_{9} b_{3} \\
& +a_{2} a_{7} a_{9} b_{6}+a_{2} a_{7} a_{11} b_{4}+a_{2} a_{7} a_{11} b_{5}+a_{2} a_{8} a_{10} b_{5} \\
& +a_{2} a_{8} a_{12} b_{2}+a_{2} a_{8} a_{12} b_{6}+a_{3} a_{4} a_{5} a_{8}+a_{3} a_{4} a_{6} a_{7} \\
& +a_{3} a_{5} a_{10} b_{1}+a_{3} a_{5} a_{10} b_{4}+a_{3} a_{5} a_{10} b_{6}+a_{3} a_{5} a_{12} b_{3} \\
& +a_{3} a_{5} a_{12} b_{5}+a_{3} a_{6} a_{9} b_{5}+a_{3} a_{6} a_{11} b_{1}+a_{3} a_{6} a_{11} b_{6} \\
& +a_{3} a_{7} a_{10} b_{2}+a_{3} a_{7} a_{10} b_{3}+a_{3} a_{7} a_{12} b_{4}+a_{3} a_{8} a_{11} b_{2} \\
& +a_{4} a_{5} a_{9} b_{2}+a_{4} a_{6} a_{10} b_{3}+a_{4} a_{6} a_{12} b_{2}+a_{4} a_{6} a_{12} b_{4} \\
& +a_{4} a_{7} a_{9} b_{1}+a_{4} a_{7} a_{9} b_{5}+a_{4} a_{7} a_{11} b_{6}+a_{4} a_{8} a_{10} b_{4} \\
& +a_{4} a_{8} a_{10} b_{6}+a_{4} a_{8} a_{12} b_{1}+a_{4} a_{8} a_{12} b_{3}+a_{4} a_{8} a_{12} b_{5} \\
& +a_{5} a_{6} a_{7} a_{8}+a_{5} a_{6} a_{9} a_{12}+a_{5} a_{6} a_{10} a_{11}+a_{5} a_{7} b_{1} b_{2} \\
& +a_{5} a_{7} b_{1} b_{3}+a_{5} a_{7} b_{2} b_{4}+a_{5} a_{7} b_{3} b_{4}+a_{6} a_{8} b_{1} b_{2} \\
& +a_{6} a_{8} b_{1} b_{4}+a_{6} a_{8} b_{2} b_{3}+a_{6} a_{8} b_{3} b_{4}+a_{7} a_{8} a_{9} a_{12} \\
& +a_{7} a_{8} a_{10} a_{11}+a_{9} a_{10} a_{11} a_{12}+a_{9} a_{11} b_{3} b_{4}+a_{9} a_{11} b_{3} b_{5} \\
& +a_{9} a_{11} b_{4} b_{6}+a_{9} a_{11} b_{5} b_{6}+a_{10} a_{12} b_{3} b_{4}+a_{10} a_{12} b_{3} b_{6} \\
& +a_{10} a_{12} b_{4} b_{5}+a_{10} a_{12} b_{5} b_{6}+b_{1} b_{2} b_{3} b_{4}+b_{1} b_{2} b_{5} b_{6} \\
& \left.+b_{3} b_{4} b_{5} b_{6}\right) \text {. }
\end{aligned}
$$

\section{References}

[1] Hamley I W 1998 The Physics of Block Copolymers (New York: Oxford University Press)

[2] Andelman D, Brochard F and Joanny J-F 1987 J. Chem. Phys. 863673

[3] Seul M and Sammon M J 1990 Phys. Rev. Lett. 641903

[4] Leibler S and Andelman D 1987 J. Physique 482013

[5] Strey R, Schomäcker R, Roux D, Nallet F and Olsson U 1990 J. Chem. Soc. Faraday Trans. 86225

[6] Minewaki K, Kato T, Yoshida H, Imai M and Ito K 2001 Langmuir 171864

[7] Matsen M W 1995 Phys. Rev. Lett. 744225

[8] Matsen M W 1995 Macromolecules 285765

[9] Janert P K and Schick M 1997 Macromolecules 303916

[10] Janert P K and Schick M 1998 Macromolecules 311109

[11] Janert P K and Schick M 1997 Macromolecules 30137

[12] Mareau V H, Akasaka S, Osaka T and Hasegawa H 2007 Macromolecules 409032

[13] Pinna M, Zvelindovsky A V, Todd S and Goldbeck-Wood G 2006 J. Chem. Phys. 125154905

[14] Honda T and Kawakatsu T 2006 Macromolecules 392340

[15] Ly D Q, Honda T, Kawakatsu T and Zvelindovsky A V 2007 Macromolecules $\mathbf{4 0} 2928$

[16] Ohta T and Ito I 1995 Phys. Rev. E 525250

[17] Netz R R, Andelman A and Schick M 1997 Phys. Rev. Lett. 791058

[18] Villain-Guillot S and Andelman D 1998 Eur. Phys. J. B 495

[19] Garel T and Doniach S 1982 Phys. Rev. B 26325

[20] Gompper G and Schick M 1990 Phys. Rev. Lett. 651116

[21] Nonomura M, Yamada K and Ohta T 2003 J. Phys.: Condens. Matter 15 L423

[22] Brazovskii S A 1975 Sov. Phys._JETP 4185

[23] Fredrickson G H and Helfand E 1987 J. Chem. Phys. 87697

[24] Matsen M W 1994 Phys. Rev. Lett. 722660 
[25] Qi S and Wang Z-G 1996 Phys. Rev. Lett. 761679

[26] Qi S and Wang Z-G 1996 Phys. Rev. E 551682

[27] Matsen M W 1998 Phys. Rev. Lett. 804470

[28] Mecke K R 1996 Phys. Rev. E 534794

[29] Sofonea V and Mecke K R 1999 Eur. Phys. J. B 899
[30] Mao Y, McLeish T C B, Teixeira P I C and Read D J 2001 Eur. Phys. J. E 669

[31] Rancon Y and Charvolin J 1988 J. Phys. Chem. 926339

[32] McGrath K M, Kékicheff P and Kléman M 1993 J. Physique II 3903 